\title{
Convolutional Hierarchical Attention Network for Query-Focused Video Summarization
}

\author{
Shuwen Xiao, ${ }^{1}$ Zhou Zhao, $^{1 *}$ Zijian Zhang, ${ }^{1}$ Xiaohui Yan, ${ }^{2}$ Min Yang ${ }^{3}$ \\ ${ }^{1}$ College of Computer Science and Technology, Zhejiang University, Hangzhou, China \\ ${ }^{2} \mathrm{CBG}$ Intelligent Engineering Dept., Huawei Technologies, China \\ ${ }^{3}$ Shenzhen Institutes of Advanced Technology (SIAT), Chinese Academy of Sciences \\ \{xiaoshuwen, zhaozhou, ckczzj\}@zju.edu.com, yanxiaohui2@ huawei.com, min.yang@ siat.ac.cn
}

\begin{abstract}
Previous approaches for video summarization mainly concentrate on finding the most diverse and representative visual contents as video summary without considering the user's preference. This paper addresses the task of query-focused video summarization, which takes user's query and a long video as inputs and aims to generate a query-focused video summary. In this paper, we consider the task as a problem of computing similarity between video shots and query. To this end, we propose a method, named Convolutional Hierarchical Attention Network (CHAN), which consists of two parts: feature encoding network and query-relevance computing module. In the encoding network, we employ a convolutional network with local self-attention mechanism and query-aware global attention mechanism to learns visual information of each shot. The encoded features will be sent to query-relevance computing module to generate queryfocused video summary. Extensive experiments on the benchmark dataset demonstrate the competitive performance and show the effectiveness of our approach.
\end{abstract}

\section{Introduction}

Recently, there is an emerging research direction in the task of video summarization, which is query-focused video summarization (Sharghi, Laurel, and Gong 2017; Zhang et al. 2018; Vasudevan et al. 2017; Wei et al. 2018). Given a long video and a user query which is some concepts, the goal of query-focused video summarization is not only to remove the redundant parts of the video, to find key frame / shots in the video, but also to pick out those segments that related to the user's query. It can be helpful in some scenarios such as when a user wants to customize a brief summary from his daily video logs.

Generic video summarization is to generate a compact version of the original video by selecting the highlights of the long video and eliminating the trivial content. Automated video summarization techniques are useful in many domains, such as motion recognition, surveillance video analysis, visual diary creation for personal lifelog videos, and video previews of video sites. Besides, modules for

\footnotetext{
${ }^{*}$ Corresponding author. Copyright (C) 2020, Association for the Advancement of Artificial Intelligence (www.aaai.org). All rights reserved.
}

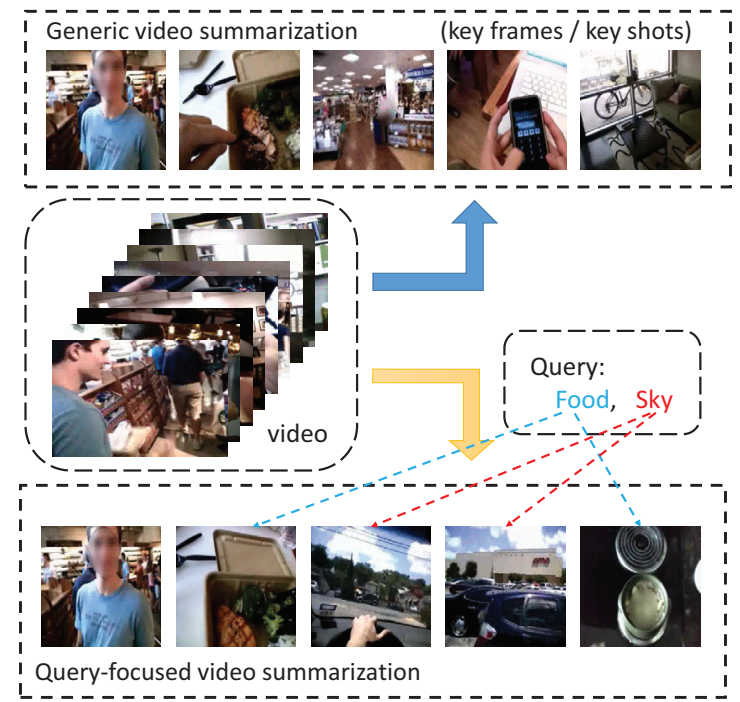

Figure 1: The goal of generic video summarization is to select a compact subset of frames or shots that remains the important information from the original video, while the task of query-focused video summarization takes user's query into consideration and generate query-related video summaries.

video summarization can also be implemented as an intermediate component to assist downstream tasks. As is shown in figure 1, there are three differences between queryfocused video summarization and generic video summarization. Firstly, the video summary needs to take the subjectivity of users into account, as different user queries may receive different video summaries. Secondly, trained video summarizers cannot meet all the users' preferences and the performance evaluation is often to measure the temporal overlap, makes it hard to capture the semantic similarity between summaries and original videos (Sharghi, Laurel, and Gong 2017). Thirdly, the textual query will bring additional semantic information to the task.

Recent researches on query-focused video summarization (Sharghi, Laurel, and Gong 2017; Zhang et al. 2018) mainly consider the task as sequence labeling problem and 
they employ models based on sequential structure such as long short-term memory (LSTM) (Hochreiter and Schmidhuber 1997). At each time step, the model outputs a binary score to predict whether this frame is important and related to the given query. However, sequential models usually perform the computation step by step and when the length of video increases, the computing time for the models also become longer. Moreover, sequential models cannot easily handle long distance relation among the video when it is too long, because of the problem of vanishing gradients. In (Rochan, Ye, and Wang 2018), they use fully convolutional networks as an encoder-decoder structure to solve video summarization task, which shows the ability of convolutional networks to generate high-quality video summaries.

In this paper, we consider the task of query-focused video summarization as a ranking problem, where we first select out the important visual content and then compute the similarity between visual content and the given query. We propose a novel method, named Convolutional Hierarchical Attention Network (CHAN), which consists of two parts: 1) a feature encoding network to learn features from each video shot in parallel from a local perspective and a global perspective; 2) query-relevance ranking module to calculate the similarity score with respect to a query for each shot and then select video content related to the given query. In feature encoding network, we utilize fully convolutional structure to decrease the dimension on the temporal sequence and the dimension of visual feature, in order to reduce the number of parameters in the model. To get a better comprehension of video, we first split video into segments and employ an information fusion layer in the encoding network, which learns visual representation inside a segment and between several segments. Combining local-level self-attention and query-aware global-level attention mechanism, information fusion layer produce features with different level information. Then, we propose a query-relevance ranking module, which can handle the modality between video and text query and compute the similarity score for each shot. Finally, the model generates query-related video summary with the similarity score.

The main contributions of this paper are summarized as follows:

- We propose a novel method named Convolutional Hierarchical Attention Network (CHAN), which is based on convolution network and global-local attention mechanism and able to generate video query-related summary in parallel.

- We present a feature encoding network to learn the features of each video shot. Inside the feature encoding network, we employ fully convolutional network with local self-attention mechanism and query-aware global attention mechanism to obtain features with more semantic information.

- We employ a query-relevance computing module which takes the feature of video shot and query as input and then calculate the similarity score. Query-related video summary is generated based on similarity score.

- We perform extensive experiments on the benchmark datasets to demonstrate the effectiveness and efficiency of our model.

The rest of this paper is organized as follows. In Section, we provide a review of the related work about generic video summarization and query-focused video summarization. In the following, Section introduces each component of our approach. A variety of experimental results are presented in Section . Finally, we provide some concluding remarks in Section .

\section{Related Works}

The goal of query-focused video summarization is obtaining a diverse subset of video frames or video segments that are not only related to the given query but also contains the original information of video. It can be categorized into two domains: (1) generic video summarization (2) query-focused video summarization.

\section{Generic Video Summarization}

Generic video summarization has been studied for many years and several methods have been proposed so far. They can be roughly categorized into three aspects: unsupervised, supervised or weakly supervised approaches.

Unsupervised video summarization approaches(Ngo, Ma, and Zhang 2003; Hong et al. 2009; Khosla et al. 2013; Zhao and Xing 2014; Panda et al. 2017) take advantage of specific selection criteria to measure the importance or interestingness of video frames and then generate video summaries. These methods have conventional methods, that is, methods that use some low-level video features for calculation, as well as more recent methods, which combine deep learning. Conventional unsupervised video summarization approaches mostly use hand-crafted heuristics (Ngo, Ma, and Zhang 2003; Zhao and Xing 2014) (such as diversity and representativeness) or frame clustering method (Otani et al. 2016; Wang, Zhu, and Gong 2016) to decide whether to choose a video frame as a keyframe. Assuming that Web images bring extra visual information which contains people's interest, some methods (Chu, Song, and Jaimes 2015; Khosla et al. 2013; Panda et al. 2017) use the web images to generate user-oriented video summaries. In (Kanehira et al. 2017), Kanehira et al. take viewpoint into consideration and calculate the video-level similarity between multiple videos to generate video summary. More recently, some methods based on deep learning have been proposed. In (Mahasseni, Lam, and Todorovic 2017), Mahasseni et al. propose a generative adversarial network. The model consists of summarizer and discriminator. The summarizer selects a subset of keyframes and then reconstruct the video and the discriminator is to minimize the distance between the information of reconstructed videos and original videos. Zhou et al. (Zhou, Qiao, and Xiang 2017) propose a reinforcement learning algorithm to summarize videos, by designing the diversityrepresentativeness reward and applying algorithm based on policy gradient to optimize the model.

Supervised video summarization methods (Gygli et al. 2014; Potapov et al. 2014; Song et al. 2015; Gygli, Grabner, and Van Gool 2015; Zhang et al. 2016; Rochan, Ye, and 
Wang 2018) have emerged in recent years, and the training data used in these methods contains raw videos and human-created ground-truth annotations. Combined with a large number of human annotations, models can capture the video content with more semantic information, so these approaches are usually superior to unsupervised methods. In (Gygli et al. 2014), video summarization is formulated as an interestingness scoring problem and frames with higher scores are selected as summaries. Some approaches utilize the additional information from web images (Khosla et al. 2013), categories (Potapov et al. 2014), and titles(Song et al. 2015) to improve the quality of video summaries. In (Gygli, Grabner, and Van Gool 2015), Gygli et al. propose an adapted submodular function with multiple objects to tackle the problem of video summarization. Yao et al.(Yao, Mei, and Rui 2016) employ a deep rank model, taking a pair of highlight segment and non-highlight segment as inputs, to rank each segment. In (Zhang et al. 2016), Zhang et al. implement the dppLSTM model by combining determinantal point process and bi-directional LSTM to predict whether a frame is a keyframe. In (Zhao, Li, and $\mathrm{Lu}$ 2018), the author proposed a hierarchical structure-adaptive RNN which is composed of two layer LSTM, where the first layer is aimed to split the video into several shots then the second layer learns shot-level information and obtain shotlevel score. In (Rochan, Ye, and Wang 2018), Rochan et al. propose a fully convolutional sequence network, of which the structure is based on convolutional layer and deconvolutional layer, consequently the network runs in parallel.

Although the supervised video summarization method performs better than the unsupervised method, there still exist some weaknesses. Firstly, getting ground-truth annotations in a video summarization dataset is time-consuming and labor-intensive. Secondly, in reality, the results returned by the video summary generator should not be unique, but the models trained with supervised methods can lead to overfitting and thus are difficult to generalize. To overcome these weaknesses, some weakly supervised methods have been proposed. These methods typically utilize readily available label, such as video categories, as additional information to improve model performance. In (Panda et al. 2017), a 3D ConvNet is trained to predict the category of the video. The importance score of each frame is obtained by calculating the back-propagated gradient returned by the true category. In (Cai et al. ), an encoder-attention-decoder structure is built, where the encoder learns the latent semantics from web videos and the decoder generate the summary.

\section{Query-Focused Video Summarization}

Compared to the generic video summarization, the task of query-focused video summarization takes user's query into account. Specifically, the dataset provides concept annotations for each shot in the video and contain more semantic information than the general form.

Some recent works take semantics into consideration when tackling the task of video summarization. In (Wei et al. 2018), they create video descriptions for videos in summarization datasets and train model based on the descriptions to leverage the additional textual information. In
(Sharghi, Gong, and Shah 2016), Sharghi et al. introduce a DPP-based algorithm, which takes user query as input and generates query-focused video summary. In (Sharghi, Laurel, and Gong 2017) explore query-focused video summarization, which generates a summary based on video content and user query and proposes a memory network based model. Vasudevan et al. (Vasudevan et al. 2017) introduce a quality-aware relevance model with submodular maximization to select important frames. In (Zhang et al. 2018), the author proposes generative adversarial network to tackle this challenge, where the model will generate a queryfocused summary, a random summary. With the ground truth summary, a three-player loss is introduced to optimize the model. These approaches are sequential model, using DPPbased algorithm or LSTM structure. To the best our knowledge, we are the first to propose an approach based on selfattention mechanism in query-focused video summarization tasks. Our proposed model is built based on convolutional neural network and attention mechanism, which can perform computation in parallel. With the query-relevance computing module, our model can produce query-related video summary.

\section{Method}

In this section, we describe the task of query-focused video summarization and illustrate the details of our proposed model.

\section{Problem Formalization}

Given a long video $\mathbf{v}$ and a query $\mathbf{q}$, the goal of queryfocused video summarization is to find out the diverse and representative subset of query-related frames or video shots. To tackle with this challenge, we denote the task as a problem of calculating the shot-query similarity. First, we denote the video as a sequence of video shots $\left(s_{1}, s_{2}, \ldots, s_{n}\right)$, where $n$ is the number of video shots. Each video shot is a small clip of the original video. Then we denote the representation of query as $h_{q}$. Moreover, in the benchmark dataset (Sharghi, Laurel, and Gong 2017), in where each query is consist of two concept $\left(c_{1}, c_{2}\right)$, we have to compute conceptrelated score for each shot and then merge two kind of score as the query-related score. Finally, based on the score, we can produce a diverse subset of video segments which can not only represent the origin video but related to the query.

Input: A long video $\mathbf{v}$ and a query $\mathbf{q}$.

Output: A diverse subset of video shots that remains the origin information of original video and each shot in the subset is related to the given query.

\section{Feature Encoding Network}

In this part, we will introduce each components of feature encoding network, as is shown in figure 2 . The main idea of our approach is that we build a encoding model which can not only capture the relation between each two shots in local-level and global-level to find out important shot in parallel, but also search for shots that related to the query. We then propose the query-relevance computing module 


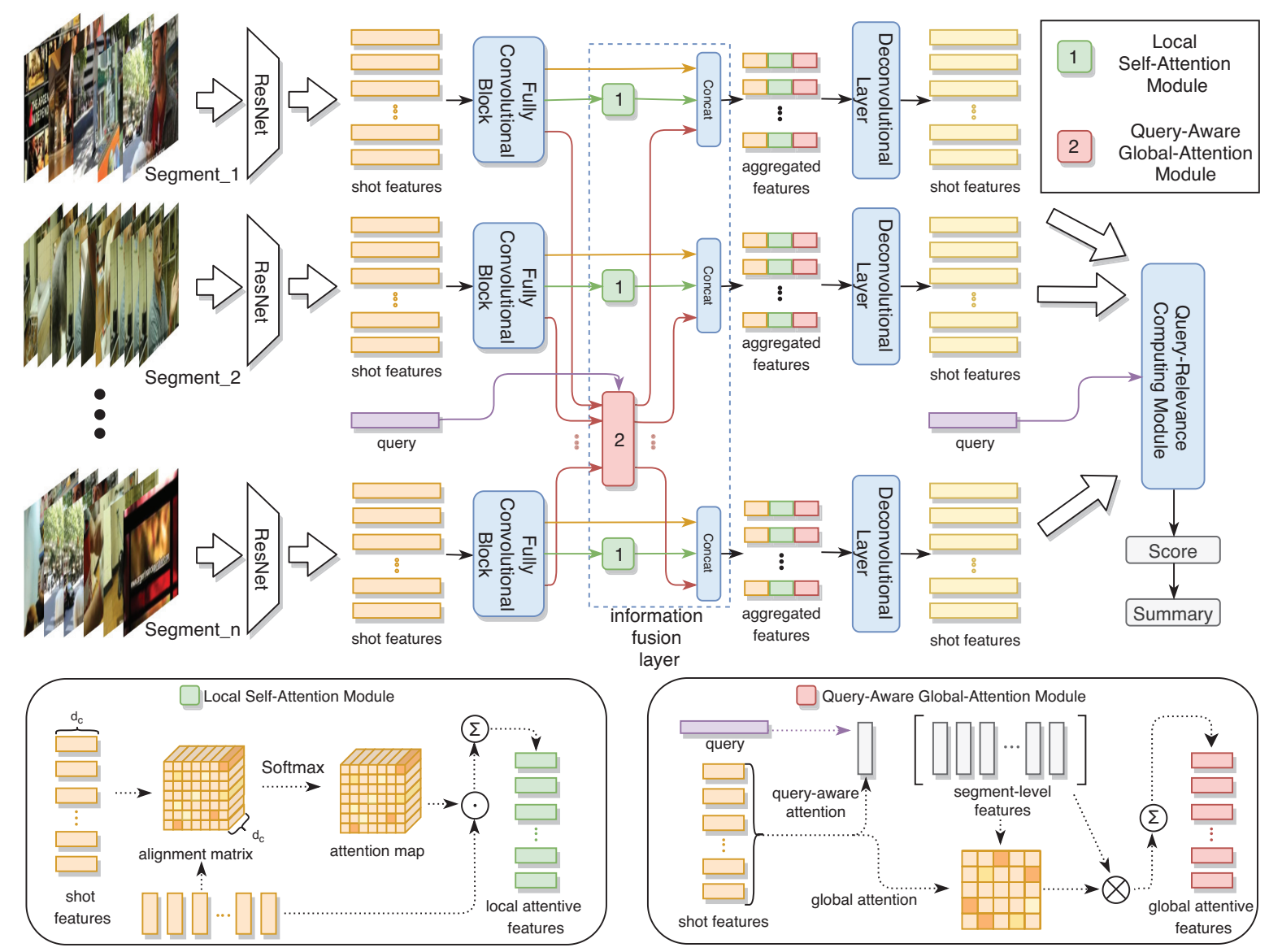

Figure 2: The framework of our proposed convolutional hierarchical attention network(CHAN). We first split the original long video into several segments and then extract visual features using pretrained network. Then we send the visual features to feature encoding network to learning shot-level features. In the feature encoding network, we propose a local self-attention module to learn the high-level semantic information in a video segment and employ a query-aware global-attention module to handle the semantic relationship between all segments and the given query. In order to reduce the parameter in the model, we employ fully convolutional block to decrease the dimension of visual feature and shorten the length of the shot sequence. Finally, all the aggregated shot-level visual features will be sent to query-relevance ranking module to obtain similarity score and the query-related video summary will be generated based on the similarity score.

to compute the similarity score between encoded shot feature and query. We use convolutional structure and attention mechanism, in which the computational processes are parallel and can capture multi-distance visual information.

Considering the hierarchy of video, a long video consists of several stories, and a story contains multiple segments, where each segment is composed of many frames. In order to get better comprehension of long videos, we need to learn the representation of video from different levels, which means we need to split the video into small video segments. There are some heuristic algorithms like uniform segmentation and the method based on visual feature (Chu, Song, and Jaimes 2015). We construct video segments using the Kernel Temporal Segmentation (Potapov et al. 2014), which preserves visual consistency in each segment.
Fully Convolutional Block After the segmentation process, we then use the pretrained ResNet to extract the visual feature of each shot. We denote the shot-level features in a specific video segment as $\left(\mathbf{v}_{1}, \mathbf{v}_{2}, \ldots, \mathbf{v}_{s_{k}}\right)$, where $s_{k}$ is the number of shots in the video segment. We propose a convolutional structure to further encode the visual feature.

To build a model with better vision of video shots in local area and parallel computation process, we use 1D fullyconvolutional network architecture (Long, Shelhamer, and Darrell 2015) to encode the shot-level visual feature. Moreover, for the purpose of handling long distance among the video segment, we utilize dilated convolutions to obtain larger receptive field. We first propose convolutional networks with different filter size and then concatenate their outputs which enables the model to receive more information. The dilated convolution operation on $i$-th shot in a 
video segment is defined as,

$$
o_{i}=\sum_{t=-k}^{k} f(t) \cdot \mathbf{v}_{i+d \cdot t}
$$

where $2 k+1$ is the filter size, $f$ is the filter and $d$ is dilation factor. With different filter sizes and large dilation, we can obtain outputs that represent more information from inputs. Then we employ a pooling layer on the temporal axis of the video, which can reduce the computing time and also decrease the running memory of the model. We connect different fully convolutional block and construct a multi-layer block to extracted representative features.

Information Fusion Layer We denote the output features of fully convolutional block as $\left(\hat{v}_{1}, \hat{v}_{2}, \ldots, \hat{v}_{t}\right)$, where $t$ is length of output feature sequence, and the output features are the inputs of the information fusion layer. The outputs of information fusion layer are a sequence of concatenated vectors of outputs from previous block, local attentive representations and query-aware global attentive representations.

The local self-attention module is designed to capture the semantic relations between all shots among a video segment. The detailed structure is presented in the left bottom of figure 2. Given the sequence of output features $\left(\hat{v}_{1}, \hat{v}_{2}, \ldots, \hat{v}_{t}\right)$ from fully convolutional block, we can compute the alignment matrix. The alignment score vector for $i$-th element and $j$-th element is given by

$$
f\left(\hat{v}_{i}, \hat{v}_{j}\right)=\mathbf{P} \tanh \left(\mathbf{W}_{1} \hat{v}_{i}+\mathbf{W}_{2} \hat{v}_{j}+\mathbf{b}\right),
$$

where $\mathbf{P}, \mathbf{W}_{1}, \mathbf{W}_{2} \in R^{d_{c} \times d_{c}}$ are the trainable parameters, $\mathbf{b} \in R^{d_{c}}$ is bias vector and $d_{c}$ is the dimension of feature vector $\hat{v}_{i}$. The score vector $f\left(\hat{v}_{i}, \hat{v}_{j}\right) \in R^{d_{c}}$ and the shape of output alignment score matrix is $t \times t \times d_{c}$, which means the module can focus on the representative features from a temporal perspective and from the viewpoint of each dimension of the features. We propose this module to learn the relative semantic relationship of different frames in the same segments, and for different segments, the relation structure should be similar. Therefore, for different video segments, our local self-attention modules share all the trainable parameters, which also reduces the amount of parameters in our model. The local attentive video feature for $i$-th element is computed by,

$$
\begin{aligned}
\gamma_{i j} & =\frac{\exp \left(f\left(\hat{v}_{i}, \hat{v}_{j}\right)\right)}{\sum_{k=0}^{t} \exp \left(f\left(\hat{v}_{i}, \hat{v}_{k}\right)\right)} \\
\hat{v}_{i}^{l} & =\sum_{j=0}^{t} \gamma_{i j} \odot \hat{v}_{j},
\end{aligned}
$$

Then we introduce the query-aware global attention module which is to model the relationship of different video segments among the video and to generate query-focused visual representation, as is shown in the right bottom of figure 2. First, given a sequence of visual representation generated from the fully convolutional block $\left(\hat{v}_{1}, \hat{v}_{2}, \ldots, \hat{v}_{t}\right)$ and a query $q$, which is composed of two concept $(c 1, c 2)$, we can calculate the query-aware segment-level visual feature for this video segment by,

$$
\begin{aligned}
e_{i} & =v^{T} \tanh \left(\mathbf{W}_{1} \hat{v}_{i}+\mathbf{W}_{2} \mathbf{h}^{q}+b\right) \\
\gamma_{i} & =\frac{\exp \left(e_{i}\right)}{\sum_{k=0}^{t} \exp \left(e_{k}\right)} \\
v^{(s)} & =\sum_{i=0}^{t} \gamma_{i} \hat{v}_{i}
\end{aligned}
$$

where $v^{T}, \mathbf{W}_{1}, \mathbf{W}_{2}$ are the trainable parameters, $b$ is bias vector and $h^{q}$ is the average of representation of concepts. The query-aware segment-level features represent aggregated representation for a video segment to a specific query. Then we compute the query-aware globalattentive representation for each shot. Given the visual feature $\hat{v}_{i}$ and all the segment-level visual representation $\left(v_{1}^{(s)}, v_{2}^{(s)}, \ldots, v_{m}^{(s)}\right)$, where $m$ is the number of video segments, the global-attentive representation for $i$-th shot can be computed by,

$$
\begin{aligned}
e_{j}^{g} & =v^{T} \tanh \left(\mathbf{W}_{1}^{g} \hat{v}_{i}+\mathbf{W}_{2}^{g} v_{j}^{(s)}+b\right) \\
\gamma_{j}^{g} & =\frac{\exp \left(e_{j}^{g}\right)}{\sum_{k=0}^{m} \exp \left(e_{k}^{g}\right)} \\
\hat{v}_{i}^{g} & =\sum_{j=0}^{m} \gamma_{j}^{g} v_{j}^{(s)}
\end{aligned}
$$

Finally, we concatenate three kinds of features as the outputs of the information fusion layer. More specifically, the fused feature for the $i$-th item is $\hat{v}_{i}^{f}=\left[\hat{v}_{i} ; \hat{v}_{i}^{l} ; \hat{v}_{i}^{g}\right]$.

Deconvolutional Layer We use fully convolutional block to decrease the number on temporal axis of video features and we propose several 1D deconvolutional layers to recover the original number of video shots.

\section{Query-Relevance Computing Module}

In this part, we are going to introduce our query-relevance computing module, which can compute the similarity score between video shot and user's query.

Due to the fact that a query is composed of two concepts, our goal is to learn a module that can select the most concept-related shots by returning a concept-relevant score. The module takes the shot-level features generated by feature encoding network, which are denoted as $\left(\hat{v}_{1}^{f}, \hat{v}_{2}^{f}, \ldots, \hat{v}_{n}^{f}\right)$ and concepts as inputs. Moreover, each segment is related to one or multiple concepts. Given a specific concept $c$, we first obtain its embedding feature $f_{c}$ using pretrained language model. Given a concept feature $f_{c}$ and shot-level features $\hat{v}_{i}^{f}$ of $i$-th shot, we first calculate their distance-based similarity by

$$
d_{i}=\mathbf{W}_{f} \hat{v}_{i}^{f} \odot \mathbf{W}_{c} f_{c}
$$

where $\mathbf{W}_{f}$ and $\mathbf{W}_{c}$ are the parameter matrices which project the visual features and textual features into the same vector space. Then we let the output pass a MLP and get the 
concept-relevant score between $i$-th segment and concept $c$. The average of two concept-relevant score is taken as the query-relevant score $s=\left\{s_{1}, s_{2}, \ldots, s_{n}\right\}$.

Given the ground truth annotations $\hat{s}=$ $\left\{\hat{s}_{1}, \hat{s}_{2}, \ldots, \hat{s}_{n}\right\} \in[0,1]$ which represents whether a shot is related to a concept, the loss can be defined as:

$$
L_{s u m m}=\frac{1}{T} \sum_{t=1}^{T} \hat{s}_{t} \log s_{t}+\left(1-\hat{s}_{t}\right) \log \left(1-s_{t}\right)
$$

By minimizing the loss, query-relevance computing module can focus on the most concept-related video shots.

\section{Experiments}

\section{Datasets and Experiment Settings}

Datasets We evaluate our method on the query-focused video summarization dataset proposed in (Sharghi, Laurel, and Gong 2017). The dataset is built upon the UT Egocentric(UTE) dataset (Lee, Ghosh, and Grauman 2012), which contains videos taken from the first person perspective. The dataset has 4 videos containing different daily life scenarios, each of which lasts 3 5 hours. In (Sharghi, Laurel, and Gong 2017), they provide a set of concepts for user's queries, in which the total number of concepts is 48 . The concepts are concise and diverse, which are related to some common objects in our daily life. Each query is composed of two concepts and there are 46 queries in the dataset. As for queries, there are four different scenarios in this task (Sharghi, Laurel, and Gong 2017), that is, 1) all concepts in the query appear in the same video shot, 2) all concepts in the query appear in the video but not in the same shot, 3) some of the concepts in the query appear in the video, 4) none of the concepts in the query appear in the video. It is worth mentioning that the fourth scenario is to some extend the same as general form video summarization. The dataset provides per-shot annotation, from which each shot is labeled with several concepts.

Data Preprocessing We preprocess the videos in queryfocused video summarization dataset as follow. To get the shot features, we sample the video to $1 \mathrm{fps}$, then reshape the size of all frames to $224 \times 224$. Next, we extract visual representation of each frame with the pretrained ResNet (Szegedy et al. 2017) which is pretrained on ImageNet (Deng et al. 2009), and taking the 2048-dimensional vector for each frame. Similar to the setting in (Sharghi, Laurel, and Gong 2017), we take 5 seconds as a shot and compute the average of each frame from a shot as its shot-level feature. Utilizing the KTS algorithm (Potapov et al. 2014), we can split video into small segments under the conditions that the number of segments in a video is no more than 20 and the number of shots in a segments is no more than 200. For the concept words in each query, we extract its word representation using publicly available Glove vectors (Pennington, Socher, and Manning 2014).
Evaluation Protocol For fair comparison, we follow the protocols setting in (Sharghi, Laurel, and Gong 2017). Considering that the video summary should be a relatively subjective result rather than a unique result, the author provides a dataset with dense per-shot annotations. They measure the performance of the model by calculating semantic similarity between different shots, rather than just measuring temporal overlap or relying on low-level visual features. In (Sharghi, Laurel, and Gong 2017), they first calculate the conceptual similarity between each two video shots based on the intersection-over-union(IOU) of their related concepts. Then they use the conceptual similarity as the edge weights in the bipartite graph between two summaries to find the maximum weight matching of the graph. Finally, precision, recall and F1 score can be computed based on the number of matching pairs.

\section{Implementation Details}

We use Pytorch to implement our approach on a server with a GTX TITAN X card. In the feature encoding layer, we propose two-layer fully convolution block, in which the output channel dimension for first layer is 256 and for the second one is 512. In the local self-attention module and queryaware global attention module, the dimension of attention $d_{c}$ is set to 256. The dimension of the visual-textual fused space in query-relevance computing module is 512. Following the setting in (Sharghi, Laurel, and Gong 2017), we randomly select two videos for training, one for testing and the remaining one for testing. In the training process, we use Adam optimizer (Kingma and $\mathrm{Ba} 2014$ ) to minimize the loss, with its initial learning rate 0.0001 and decay rate of 0.8 . The minibatch strategy is also used and the batch size is set to 5. After obtaining the similarity score, we create query-related video summary by selecting video shots with the highest score.

\section{Compared Models}

In our evaluation, we compare our proposed method with other query-focused video summarization approaches as follows:

- SeqDPP method (Gong et al. 2014) which formulates video summarization as a subset selection problem and use submodular maximization to found a good summary. The original approach does not consider user queries.

- SH-DPP (Sharghi, Gong, and Shah 2016) is the extension of SeqDPP method, where the author add a extra layer in the process of SeqDPP to judge whether a video shot is related to a given query.

- QC-DPP (Sharghi, Laurel, and Gong 2017) is the extension of SeqDPP method. In this approach, the author introduces memory network to parameterize the kernel matrix.

- TPAN (Zhang et al. 2018) is three-player adversarial network. The author uses generative adversarial network to tackle with the task and introduce a random summary as an extra adversarial sample. 
Table 1: Comparison results on the query-focused video summarization dataset in terms of Precision, Recall and F1-score.

\begin{tabular}{|c|c|c|c|c|c|c|c|c|c|c|c|c|c|c|c|}
\hline & \multicolumn{3}{|c|}{ SeqDPP } & \multicolumn{3}{|c|}{ SH-DPP } & \multicolumn{3}{|c|}{ QC-DPP } & \multicolumn{3}{|c|}{ TPAN } & \multicolumn{3}{|c|}{ CHAN } \\
\hline & Pre & Rec & F1 & Pre & $\operatorname{Rec}$ & F1 & Pre & $\operatorname{Rec}$ & F1 & Pre & $\operatorname{Rec}$ & F1 & Pre & $\operatorname{Rec}$ & F1 \\
\hline Vid1 & 53.43 & 29.81 & 36.59 & 50.56 & 29.64 & 35.67 & 49.86 & 53.38 & 48.68 & 49.66 & 50.91 & 48.74 & 54.73 & 46.57 & 49.14 \\
\hline Vid2 & 44.05 & 46.65 & 43.67 & 42.13 & 46.81 & 42.72 & 33.71 & 62.09 & 41.66 & 43.02 & 48.73 & 45.30 & 45.92 & 50.26 & 46.53 \\
\hline Vid3 & 49.25 & 17.44 & 25.26 & 51.92 & 29.24 & 36.51 & 55.16 & 62.40 & 56.47 & 58.73 & 56.49 & 56.51 & 59.75 & 64.53 & 58.65 \\
\hline Vid4 & 11.14 & 63.49 & 18.15 & 11.51 & 62.88 & 18.62 & 21.39 & 63.12 & 29.96 & 36.70 & 35.96 & 33.64 & 25.23 & 51.16 & 33.42 \\
\hline Avg. & 39.47 & 39.35 & 30.92 & 39.03 & 42.14 & 33.38 & 40.03 & 60.25 & 44.19 & 47.03 & 48.02 & 46.05 & 46.40 & 53.13 & 46.94 \\
\hline
\end{tabular}

Table 2: Ablation analysis on query-conditioned video summarization in terms of Precision, Recall and F1-score.

\begin{tabular}{l||c|c|c}
\hline Model & Pre & Rec & F1 \\
\hline CHAN w/o Local Att & 42.72 & 49.04 & 43.26 \\
CHAN w/o Global Att & 37.62 & 43.17 & 38.09 \\
\hline CHAN & 46.40 & 53.13 & 46.94 \\
\hline
\end{tabular}

\section{Experimental Results}

Table 1 show the comparison results for query-focused video summarization in terms of precision, recall and f1score. We compare our method with other approaches that have been used in this task. It is shown that our method outperforms the state-of-the-art approach (TPAN) by $1.9 \%$. More specifically, for the video 2 and video 3, we obtain a better performance than TPAN (Zhang et al. 2018), by $2.64 \%$ and $3.6 \%$ respectively. The improvements of performance identify the effectiveness of our approaches to learn the relevance between the video shots and user's query. The average running time for inference phase of each video is $134.4 \mathrm{~ms}$, which is shorter than $1.614 \mathrm{~s}$ (Zhang et al. 2018) by $91.6 \%$, showing the efficiency of our approach for its parallel computing ability.

The ablation study of each components in our model, which is shown in Table 2. The F1-score of the model without local self-attention module is reduced by $7.84 \%$. Moreover, without query-aware global attention module, the performance decreases by $18.8 \%$. The results illustrate the effectiveness of these two attention modules. Local selfattention module and query-aware global attention module can capture visual information inside a video segment and between segments, which is helpful to improve performance.

\section{Qualitative Results}

We present the visualization result in Figure 3. We take one user queries, "Book Street", as the inputs of our model. The $\mathrm{x}$-axis in the figure represents the temporal order of video shots. The green lines represent the ground truth annotations of key shots which are related to the query. The blue lines denote the results of predicted key shots. It can be observed that the selected summaries are related to one or both concepts in the given query, which demonstrates that our proposed method is able to find diverse, representative and query-related summaries.

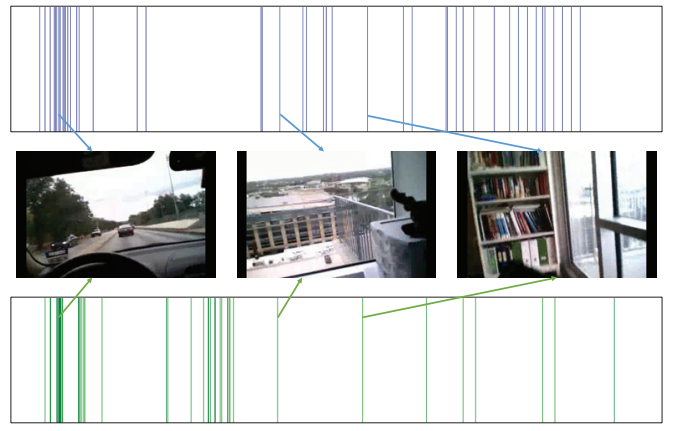

(a) Visualization Result (Book, Street)

Figure 3: The visualization results of our approach. The $\mathrm{x}$ axis denotes the video shot number. Green lines represent the ground truth annotations and blue lines represent the predicted key shots from our method. Figures shows the summary for the query "Book Street"

\section{Conclusion}

In this paper, we study the task of query-focused video summarization. We formulate query-focused video summarization as a task of computing relevance score between video shot and query, then we generate video summary based on the relevance score. We propose a Convolutional Hierarchical Attention Network which firstly encodes the video shots in parallel, then computes relevance scores between each shot and query and finally generate query-related video summary. Extensive experiments on the benchmark dataset show the effectiveness and efficiency of our approach. Moving forward, we are going to design a more general model which can generate video summary for new queries.

\section{Acknowledgments}

This work is supported in part by the National Natural Science Foundation of China under Grants No.61602405, No.U1611461, No.61751209, No.61836002 and China Knowledge Centre for Engineering Sciences, and Technology.

\section{References}

Cai, S.; Zuo, W.; Davis, L. S.; and Zhang, L. Weaklysupervised video summarization using variational encoderdecoder and web prior.

Chu, W.-S.; Song, Y.; and Jaimes, A. 2015. Video 
co-summarization: Video summarization by visual cooccurrence. In CVPR, 3584-3592. IEEE.

Deng, J.; Dong, W.; Socher, R.; Li, L.-J.; Li, K.; and FeiFei, L. 2009. Imagenet: A large-scale hierarchical image database. In Computer Vision and Pattern Recognition, 2009. CVPR 2009. IEEE Conference on, 248-255. Ieee.

Gong, B.; Chao, W.-L.; Grauman, K.; and Sha, F. 2014. Diverse sequential subset selection for supervised video summarization. In Advances in Neural Information Processing Systems, 2069-2077.

Gygli, M.; Grabner, H.; Riemenschneider, H.; and Van Gool, L. 2014. Creating summaries from user videos. In $E C C V$, 505-520. Springer.

Gygli, M.; Grabner, H.; and Van Gool, L. 2015. Video summarization by learning submodular mixtures of objectives. In Proceedings of the IEEE Conference on Computer Vision and Pattern Recognition, 3090-3098.

Hochreiter, S., and Schmidhuber, J. 1997. Long short-term memory. Neural computation 9(8):1735-1780.

Hong, R.; Tang, J.; Tan, H.-k.; Yan, S.; Ngo, C.-w.; and Chua, T.-s. 2009. Event driven summarization for web videos. In ACM Multimedia 1st Workshop on Social Media, ACM-MM-WSM. Citeseer.

Kanehira, A.; Van Gool, L.; Ushiku, Y.; and Harada, T. 2017. Viewpoint-aware video summarization. Proceedings of the Computer Vision and Pattern Recognition, Honolulu, HI, USA 21-26.

Khosla, A.; Hamid, R.; Lin, C.-J.; and Sundaresan, N. 2013. Large-scale video summarization using web-image priors. In Proceedings of the IEEE conference on computer vision and pattern recognition, 2698-2705.

Kingma, D. P., and Ba, J. 2014. Adam: A method for stochastic optimization. arXiv preprint arXiv:1412.6980.

Lee, Y. J.; Ghosh, J.; and Grauman, K. 2012. Discovering important people and objects for egocentric video summarization. In Computer Vision and Pattern Recognition (CVPR), 2012 IEEE Conference on, 1346-1353. IEEE.

Long, J.; Shelhamer, E.; and Darrell, T. 2015. Fully convolutional networks for semantic segmentation. In Proceedings of the IEEE conference on computer vision and pattern recognition, 3431-3440.

Mahasseni, B.; Lam, M.; and Todorovic, S. 2017. Unsupervised video summarization with adversarial lstm networks. In $C V P R, 2982-2991$. IEEE.

Ngo, C.-W.; Ma, Y.-F.; and Zhang, H.-J. 2003. Automatic video summarization by graph modeling. In Computer $\mathrm{Vi}$ sion, 2003. Proceedings. Ninth IEEE International Conference on, 104-109. IEEE.

Otani, M.; Nakashima, Y.; Rahtu, E.; Heikkilä, J.; and Yokoya, N. 2016. Video summarization using deep semantic features. In $A C C V, 361-377$. Springer.

Panda, R.; Das, A.; Wu, Z.; Ernst, J.; and Roy-Chowdhury, A. K. 2017. Weakly supervised summarization of web videos. In $I C C V, 3677-3686$. IEEE.
Pennington, J.; Socher, R.; and Manning, C. 2014. Glove: Global vectors for word representation. In Proceedings of the 2014 conference on empirical methods in natural language processing (EMNLP), 1532-1543.

Potapov, D.; Douze, M.; Harchaoui, Z.; and Schmid, C. 2014. Category-specific video summarization. In $E C C V$, 540-555. Springer.

Rochan, M.; Ye, L.; and Wang, Y. 2018. Video summarization using fully convolutional sequence networks. $\operatorname{arXiv}$ preprint arXiv:1805.10538.

Sharghi, A.; Gong, B.; and Shah, M. 2016. Query-focused extractive video summarization. In European Conference on Computer Vision, 3-19. Springer.

Sharghi, A.; Laurel, J. S.; and Gong, B. 2017. Queryfocused video summarization: Dataset, evaluation, and a memory network based approach. In The IEEE Conference on Computer Vision and Pattern Recognition (CVPR), 2127-2136.

Song, Y.; Vallmitjana, J.; Stent, A.; Jaimes, A.; et al. 2015. Tvsum: Summarizing web videos using titles. In CVPR, 5179-5187. IEEE.

Szegedy, C.; Ioffe, S.; Vanhoucke, V.; and Alemi, A. A. 2017. Inception-v4, inception-resnet and the impact of residual connections on learning. In $A A A I$, volume 4, 12.

Vasudevan, A. B.; Gygli, M.; Volokitin, A.; and Van Gool, L. 2017. Query-adaptive video summarization via qualityaware relevance estimation. In Proceedings of the 2017 ACM on Multimedia Conference, 582-590. ACM.

Wang, J.; Zhu, X.; and Gong, S. 2016. Video semantic clustering with sparse and incomplete tags. In AAAI, 36183624.

Wei, H.; Ni, B.; Yan, Y.; Yu, H.; Yang, X.; and Yao, C. 2018. Video summarization via semantic attended networks. In AAAI.

Yao, T.; Mei, T.; and Rui, Y. 2016. Highlight detection with pairwise deep ranking for first-person video summarization. In The IEEE Conference on Computer Vision and Pattern Recognition (CVPR).

Zhang, K.; Chao, W.-L.; Sha, F.; and Grauman, K. 2016. Video summarization with long short-term memory. In European conference on computer vision, 766-782. Springer.

Zhang, Y.; Kampffmeyer, M.; Liang, X.; Tan, M.; and Xing, E. P. 2018. Query-conditioned three-player adversarial network for video summarization. arXiv preprint arXiv:1807.06677.

Zhao, B., and Xing, E. P. 2014. Quasi real-time summarization for consumer videos. In CVPR, 2513-2520.

Zhao, B.; Li, X.; and Lu, X. 2018. Hsa-rnn: Hierarchical structure-adaptive rnn for video summarization. In Proceedings of the IEEE Conference on Computer Vision and Pattern Recognition, 7405-7414.

Zhou, K.; Qiao, Y.; and Xiang, T. 2017. Deep reinforcement learning for unsupervised video summarization with diversity-representativeness reward. 